\title{
Multilinear Singular Integral Operators on Generalized Weighted Morrey Spaces
}

\author{
Yue Hu, ${ }^{1}$ Zhang $\mathrm{Li}^{2}{ }^{2}$ and Yueshan Wang ${ }^{3}$ \\ ${ }^{1}$ College of Mathematics and Informatics, Henan Polytechnic University, Jiaozuo 454003, China \\ ${ }^{2}$ College of Computer Science and Technology, Henan Polytechnic University, Jiaozuo 454003, China \\ ${ }^{3}$ Department of Mathematics, Jiaozuo University, Jiaozuo 454003, China
}

Correspondence should be addressed to Yue Hu; huu3y3@163.com

Received 24 April 2014; Accepted 16 June 2014; Published 17 July 2014

Academic Editor: Henryk Hudzik

Copyright (c) 2014 Yue Hu et al. This is an open access article distributed under the Creative Commons Attribution License, which permits unrestricted use, distribution, and reproduction in any medium, provided the original work is properly cited.

The purpose of this paper is to discuss the boundedness properties of multilinear Calderón-Zygmund operator and its commutator on the generalized weighted Morrey spaces.

\section{Introduction and Results}

Multilinear Calderón-Zygmund theory is a natural generalization of the linear case. The initial work on the class of multilinear Calderón-Zygmund operators was done by Coifman and Meyer in [1] and was later systematically studied by Grafakos and Torres in [2-4]. In 2009, the weighted estimates of multilinear Calderón-Zygmund singular integral operator and its commutator were established in [5] by Lerner et al. In 2013, the results of [5] were extended to the weighted Morrey space (see $[6,7])$. In this paper, we will discuss the boundedness properties of multilinear Calderón-Zygmund operator and its commutator on the generalized weighted Morrey spaces.

Let $\mathbb{R}^{n}$ be the $n$-dimensional Euclidean space, and let $\left(\mathbb{R}^{n}\right)^{m}=\mathbb{R}^{n} \times \cdots \times \mathbb{R}^{n}$ be the $m$-fold product space $(m \in \mathbb{N})$. We denote by $\mathcal{S}\left(\mathbb{R}^{n}\right)$ the space of all Schwartz functions on $\mathbb{R}^{n}$ and denote by $\mathcal{S}^{\prime}\left(\mathbb{R}^{n}\right)$ its dual space of the set of all tempered distributions on $\mathbb{R}^{n}$.

We say that a locally integrable function $K\left(y_{0}, y_{1}, \ldots, y_{m}\right)$ defined away from the diagonal $y_{0}=y_{1}=\cdots=y_{m}$ in $\left(\mathbb{R}^{n}\right)^{m+1}$ is a kernel in the class $m-C Z K(A, \epsilon)$ if it satisfies the size estimate

$$
\left|K\left(y_{0}, y_{1}, \ldots, y_{m}\right)\right| \leq \frac{A}{\left(\sum_{k, l=0}^{m}\left|y_{l}-y_{k}\right|\right)^{m n}}
$$

for some $A>0$ and all $\left(y_{0}, y_{1}, \ldots, y_{m}\right) \in\left(\mathbb{R}^{n}\right)^{m+1}$ with $y_{l} \neq y_{k}$ for some $l, k$. Moreover, assume that for some $\epsilon>0$ and all $0 \leq j \leq m$ it satisfies the smoothness estimates

$$
\begin{aligned}
& \left|K\left(y_{0}, \ldots, y_{j}, \ldots, y_{m}\right)-K\left(y_{0}, \ldots, y_{j}^{\prime}, \ldots, y_{m}\right)\right| \\
& \quad \leq \frac{A\left|y_{j}-y_{j}^{\prime}\right|^{\epsilon}}{\left(\sum_{k, l=0}^{m}\left|y_{l}-y_{k}\right|\right)^{m n+\epsilon}}
\end{aligned}
$$

whenever

$$
\left|y_{j}-y_{j}^{\prime}\right| \leq \frac{1}{2} \max _{0 \leq k \leq m}\left|y_{j}-y_{k}\right|
$$

Let $T$ be a multilinear operator initially defined on the $m$ fold product of Schwartz spaces, and, by taking values into the space of tempered distributions,

$$
T: \mathcal{S}\left(\mathbb{R}^{n}\right) \times \cdots \times \mathcal{S}\left(\mathbb{R}^{n}\right) \longrightarrow \mathcal{S}^{\prime}\left(\mathbb{R}^{n}\right)
$$

Following [2], we say that $T$ is an $m$-linear CalderónZygmund operator if, for some $q_{1}, \ldots, q_{m} \in[1, \infty)$ and $q \in(0, \infty)$ with $1 / q=\sum_{i=1}^{m} 1 / q_{i}$, it extends to a bounded multilinear operator from $L^{q_{1}}\left(\mathbb{R}^{n}\right) \times \cdots L^{q_{m}}\left(\mathbb{R}^{n}\right)$ into $L^{q}\left(\mathbb{R}^{n}\right)$ and if there exists a kernel function $K\left(x, y_{1}, \ldots, y_{m}\right)$ in 
the class $m-C Z K(A, \epsilon)$, defined away from the diagonal $x=$ $y_{1}=\cdots=y_{m}$ in $\left(\mathbb{R}^{n}\right)^{m+1}$, satisfying

$$
\begin{aligned}
T & (\vec{f})(x) \\
& =T\left(f_{1}, \ldots, f_{m}\right)(x) \\
& =\int_{\left(\mathbb{R}^{n}\right)^{m}} K\left(x, y_{1}, \ldots, y_{m}\right) f_{1}\left(y_{1}\right) \cdots f\left(y_{m}\right) d y_{1} \cdots d y_{m}
\end{aligned}
$$

for all $x \notin \bigcap_{j=1}^{m} \operatorname{supp} f_{j}$ and $f_{1}, \ldots, f_{m}$ are $C^{\infty}$ functions with compact support.

Theorem 1 (see [5]). Let $m \geq 2$ and let $T$ be an $m$-linear Calderón-Zygmund operator. Suppose that $1 / p=\sum_{i=1}^{m} 1 / p_{i}$, $\vec{\omega}=\left(\omega_{1}, \ldots, \omega_{m}\right)$ satisfies the $A_{\vec{p}}$ condition, and $\nu_{\vec{\omega}}=$ $\prod_{i=1}^{m} \omega_{i}^{p / p_{i}}$.

(1) If $1<p_{i}<\infty, i=1, \ldots, m$, then

$$
\|T(\vec{f})\|_{L^{p}\left(v_{\vec{\omega}}\right)} \leq C \prod_{i=1}^{m}\left\|f_{i}\right\|_{L^{p_{i}}\left(\omega_{i}\right)} .
$$

(2) If $1 \leq p_{i}<\infty, i=1, \ldots, m$, and at least one of the $p_{i}=1$, then

$$
\|T(\vec{f})\|_{W L^{p}\left(v_{\vec{\omega}}\right)} \leq C \prod_{i=1}^{m}\left\|f_{i}\right\|_{L^{p_{i}}\left(\omega_{i}\right)} .
$$

The purpose of this paper is to discuss the boundedness properties of $m$-linear Calderón-Zygmund operator and its commutator on the generalized weighted Morrey spaces $M_{\varphi}^{p}(\omega)$.

The classical Morrey spaces $L^{p, \lambda}$ were originally introduced by Morrey in [8] to study the local behavior of solutions to the second-order elliptic partial differential equations. For the boundedness of the Hardy-Littlewood maximal operator, the fractional integral operator, and the CalderónZygmund singular integral operator on these spaces, we refer the readers to [9-11]. Moreover, various Morrey spaces are defined in the process of study. Mizuhara [12] introduced the generalized Morrey space $L^{p, \varphi}$; Komori and Shirai [13] defined the weighted Morrey spaces $L^{p, \kappa}(\omega)$; Guliyev [14] gave a concept of generalized weighted Morrey space $M_{\varphi}^{p}(\omega)$ which could be viewed as extension of both $L^{p, \varphi}$ and $L^{p, \kappa}(\omega)$. The boundedness of some operators on these Morrey spaces can be seen in [12-16].

Our first result can be formulated as follows.

Theorem 2. Let $m \geq 2$, let $T$ be an m-linear CalderónZygmund operator, and let $\vec{\varphi}_{k}=\left(\varphi_{k 1}, \ldots, \varphi_{k m}\right), k=1,2$. Suppose that $1 / p=\sum_{i=1}^{m} 1 / p_{i}, \vec{\omega}=\left(\omega_{1}, \ldots, \omega_{m}\right)$ satisfies the $A_{\vec{p}}$ condition with $\omega_{1}, \ldots, \omega_{m} \in A_{\infty}$, and $\left(\vec{\varphi}_{1}, \vec{\varphi}_{2}\right)$ satisfies the condition

$$
\begin{aligned}
& \int_{s}^{\infty} \frac{\text { ess } \inf _{r<t<\infty} \prod_{i=1}^{m} \varphi_{1 i}(x, t) \omega_{i}(B(x, t))^{1 / p_{i}}}{\prod_{i=1}^{m} \omega_{i}(B(x, r))^{1 / p_{i}}} \frac{d r}{r} \\
& \quad \leq C \varphi_{2}(x, s) .
\end{aligned}
$$

(1) If $1<p_{i}<\infty, i=1, \ldots, m$, then

$$
\|T(\vec{f})\|_{M_{\varphi_{2}}^{p}\left(v_{\vec{\omega}}\right)} \leq C \prod_{i=1}^{m}\left\|f_{i}\right\|_{M_{\varphi_{1 i}}^{p_{i}}\left(\omega_{i}\right)} .
$$

(2) If $1 \leq p_{i}<\infty, i=1, \ldots, m$, and at least one of the $p_{i}=1$, then

$$
\|T(\vec{f})\|_{W M_{\varphi_{2}}^{p}\left(v_{\vec{\omega}}\right)} \leq C \prod_{i=1}^{m}\left\|f_{i}\right\|_{M_{\varphi_{1 i}}^{p_{i}}\left(\omega_{i}\right)},
$$

$$
\text { where } \varphi_{2}=\prod_{i=1}^{m} \varphi_{2 i} \text { and } \nu_{\vec{\omega}}=\prod_{i=1}^{m} \omega_{i}^{p / p_{i}} \text {. }
$$

Given a locally integrable vector function $\vec{b}=\left(b_{1}, \ldots\right.$, $\left.b_{m}\right) \in(B M O)^{m}$, the commutator of $\vec{b}$ and the $m$-linear Calderón-Zygmund operator $T$, denoted here by $T_{\Sigma b}$, was introduced in [5] and is defined via

$$
T_{\Sigma b}(\vec{f})=\sum_{i=1}^{m} T_{b_{i}}^{i}(\vec{f}),
$$

where

$$
T_{b_{i}}^{i}(\vec{f})=b_{i} T(\vec{f})-T\left(f_{1}, \ldots, b_{i} f_{i}, \ldots, f_{m}\right) .
$$

And the iterated commutator $T_{\Pi b}$ was introduced in [17] and is defined by

$$
T_{\Pi b}(\vec{f})=\left[b_{1}, \ldots,\left[b_{m-1},\left[b_{m}, T\right]_{m}\right]_{m-1} \cdots\right]_{1}(\vec{f}) .
$$

To clarify the notation, if $T$ is associated in the usual way with a Calderón-Zygmund kernel $K$, then, at a formal level,

$$
\begin{array}{r}
T_{\Sigma b}(\vec{f})(x)=\int_{\left(\mathbb{R}^{n}\right)^{m}} \sum_{i=1}^{m}\left(b_{i}(x)-b_{i}\left(y_{i}\right)\right) K\left(x, y_{1}, \ldots, y_{m}\right) \\
\quad \times f_{1}\left(y_{1}\right) \cdots f_{m}\left(y_{m}\right) d y_{1} \cdots d y_{m}, \\
T_{\Pi b}(\vec{f})(x)=\int_{\left(\mathbb{R}^{n}\right)^{m}} \prod_{i=1}^{m}\left(b_{i}(x)-b_{i}\left(y_{i}\right)\right) K\left(x, y_{1}, \ldots, y_{m}\right) \\
\times f_{1}\left(y_{1}\right) \cdots f_{m}\left(y_{m}\right) d y_{1} \cdots d y_{m} .
\end{array}
$$

For $T$ is an $m$-linear Calderón-Zygmund operator, $\vec{\omega} \epsilon$ $A_{\vec{p}}$, and $\nu_{\vec{\omega}}=\prod_{i=1}^{m} \omega_{i}^{p / p_{i}}$ with $1 / p=1 / p_{1}+\cdots+1 / p_{m}$ and $\vec{b} \in(B M O)^{m}$, Lerner et al. [5] proved that $T_{\Sigma b}$ bounded from $L^{p_{1}}\left(\omega_{1}\right) \times \cdots \times L^{p_{m}}\left(\omega_{m}\right)$ to $L^{p}\left(\nu_{\vec{\omega}}\right)$ and Pérez et al. [17] extended the result to $T_{\Pi b}$. Our second result is to extend their results to the generalized weighted Morrey space.

Theorem 3. Let $m \geq 2$ and let $T$ be an $m$-linear CalderónZygmund operator. Suppose that $p_{1}, \ldots, p_{m} \in(1, \infty), p \in$ $(0, \infty)$ with $1 / p=\sum_{i=1}^{m} 1 / p_{i}, \vec{\omega}=\left(\omega_{1}, \ldots, \omega_{m}\right)$ satisfies the $A_{\vec{p}}$ condition with $\omega_{1}, \ldots, \omega_{m} \in A_{\infty}$, and $\left(\vec{\varphi}_{1}, \vec{\varphi}_{2}\right)$ satisfies the condition

$$
\begin{aligned}
& \int_{s}^{\infty}\left(1+\ln \frac{r}{s}\right)^{m} \frac{\operatorname{ess~inf}_{r<t<\infty} \prod_{i=1}^{m} \varphi_{1 i}(x, t) \omega_{i}(B(x, t))^{1 / p_{i}}}{\prod_{i=1}^{m} \omega_{i}(B(x, r))^{1 / p_{i}}} \frac{d r}{r} \\
& \leq C \varphi_{2}(x, s) .
\end{aligned}
$$


If $b_{i} \in B M O, \vec{b}=\left(b_{1}, \ldots, b_{m}\right)$, then there exist constants $C>0$ independent of $\vec{f}=\left(f_{1}, \ldots, f_{m}\right)$ such that

$$
\begin{aligned}
& \left\|T_{\Sigma b}(\vec{f})\right\|_{M_{\varphi_{2}}^{p}\left(v_{\vec{\omega}}\right)} \leq C \prod_{i=1}^{m}\left\|b_{i}\right\|_{*} \cdot \prod_{i=1}^{m}\left\|f_{i}\right\|_{M_{\varphi_{1 i}}^{p_{i}}\left(\omega_{i}\right)}, \\
& \left\|T_{\Pi b}(\vec{f})\right\|_{M_{\varphi_{2}}^{p}\left(v_{\vec{\omega}}\right)} \leq C \prod_{i=1}^{m}\left\|b_{i}\right\|_{*} \cdot \prod_{i=1}^{m}\left\|f_{i}\right\|_{M_{\varphi_{1 i}}^{p_{i}}\left(\omega_{i}\right)},
\end{aligned}
$$

where $\nu_{\vec{\omega}}=\prod_{i=1}^{m} \omega_{i}^{p / p_{i}}$ and $\varphi_{2}=\prod_{i=1}^{m} \varphi_{2 i}$.

Example 4. Let $\varphi_{1 i}(x, t)=\varphi_{2 i}(x, t)=\omega_{i}(B(x, t))^{(\kappa-1) / p_{i}}$ and $\omega_{i} \in A_{\infty}$ for $i=1, \ldots, m, 0<\kappa<1$. Then, $\left(\vec{\varphi}_{1}, \vec{\varphi}_{2}\right)$ satisfies conditions (8) and (15).

In fact, from (34), in Section 2, we have a constant $\delta>0$ such that

$$
\omega_{i}\left(B\left(x, 2^{k} s\right)\right) \geq C 2^{\delta n k} \omega_{i}(B(x, s))
$$

for all $i$. Then,

$$
\begin{aligned}
& \int_{s}^{\infty} \frac{\operatorname{ess} \inf _{r<t<\infty} \prod_{i=1}^{m} \varphi_{1 i}(x, t) \omega_{i}(B(x, t))^{1 / p_{i}}}{\prod_{i=1}^{m} \omega_{i}(B(x, r))^{1 / p_{i}}} \frac{d r}{r} \\
& \leq \int_{s}^{\infty}\left(1+\ln \frac{r}{s}\right)^{m} \\
& \quad \times \frac{\operatorname{ess~inf}{ }_{r<t<\infty} \prod_{i=1}^{m} \varphi_{1 i}(x, t) \omega_{i}(B(x, t))^{1 / p_{i}}}{\prod_{i=1}^{m} \omega_{i}(B(x, r))^{1 / p_{i}}} \frac{d r}{r} \\
& =\sum_{k=0}^{\infty} \int_{2^{k} s}^{2^{k+1} s}\left(1+\ln \frac{r}{s}\right)^{m} \prod_{i=1}^{m} \omega_{i}(B(x, r))^{(\kappa-1) / p_{i}} \frac{d r}{r} \\
& \leq C \sum_{k=0}^{\infty} k^{m} \prod_{i=1}^{m} \omega_{i}\left(B\left(x, 2^{k+1} s\right)\right)^{(\kappa-1) / p_{i}} \\
& \leq C \sum_{k=0}^{\infty} k^{m} 2^{(k+1) n \delta((\kappa-1) / p)} \prod_{i=1}^{m} \omega_{i}(B(x, s))^{(\kappa-1) / p_{i}} \\
& \leq C \prod_{i=1}^{m} \omega_{i}(B(x, s))^{(\kappa-1) / p_{i}}=C \prod_{i=1}^{m} \varphi_{2 i}(x, s) .
\end{aligned}
$$

Hence, we get the following corollaries.

Corollary 5 (see [6]). Let $m \geq 2$ and let $T$ be an $m$-linear Calderón-Zygmund operator. Suppose that $1 / p=\sum_{i=1}^{m} 1 / p_{i}$, $0<\kappa<1, \vec{\omega}=\left(\omega_{1}, \ldots, \omega_{m}\right)$ satisfies the $A_{\vec{p}}$ condition with $\omega_{1}, \ldots, \omega_{m} \in A_{\infty}$, and $\nu_{\vec{\omega}}=\prod_{i=1}^{m} \omega_{i}^{p / p_{i}}$.

(1) If $1<p_{i}<\infty, i=1, \ldots, m$, then

$$
\|T(\vec{f})\|_{L^{p, \kappa}\left(v_{\vec{\omega}}\right)} \leq C \prod_{i=1}^{m}\left\|f_{i}\right\|_{L^{p_{i}, \kappa}\left(\omega_{i}\right)} .
$$

(2) If $1 \leq p_{i}<\infty, i=1, \ldots, m$, and at least one of the $p_{i}=1$, then

$$
\|T(\vec{f})\|_{W L^{p, \kappa}\left(v_{\vec{\omega}}\right)} \leq C \prod_{i=1}^{m}\left\|f_{i}\right\|_{L^{p_{i}, \kappa}\left(\omega_{i}\right)} .
$$

Corollary 6. Let $m \geq 2$ and let $T$ be an $m$-linear CalderónZygmund operator. Suppose that $1 / p=\sum_{i=1}^{m} 1 / p_{i}, 0<\kappa<1$, $\vec{\omega}=\left(\omega_{1}, \ldots, \omega_{m}\right)$ satisfies the $A_{\vec{p}}$ condition with $\omega_{1}, \ldots, \omega_{m} \in$ $A_{\infty}$, and $\nu_{\vec{\omega}}=\prod_{i=1}^{m} \omega_{i}^{p / p_{i}}$. If $b_{i} \in B M O, \vec{b}=\left(b_{1}, \ldots, b_{m}\right)$, then there exist constants $C>0$ independent of $\vec{f}=\left(f_{1}, \ldots, f_{m}\right)$ such that

$$
\begin{aligned}
& \left\|T_{\Sigma b}(\vec{f})\right\|_{L^{p, \kappa}\left(v_{\vec{\omega}}\right)} \leq C \prod_{i=1}^{m}\left\|b_{i}\right\|_{*} \cdot \prod_{i=1}^{m}\left\|f_{i}\right\|_{L^{p_{i}, \kappa}\left(\omega_{i}\right)}, \\
& \left\|T_{\Pi b}(\vec{f})\right\|_{L^{p, \kappa}\left(v_{\vec{\omega}}\right)} \leq C \prod_{i=1}^{m}\left\|b_{i}\right\|_{*} \cdot \prod_{i=1}^{m}\left\|f_{i}\right\|_{L^{p_{i}, \kappa}\left(\omega_{i}\right)} \cdot
\end{aligned}
$$

\section{Definitions and Preliminaries}

A weight $\omega$ is a nonnegative, locally integrable function on $\mathbb{R}^{n}$. Let $B=B\left(x_{0}, r_{B}\right)$ denote the ball with the center $x_{0}$ and radius $r_{B}$. For any ball $B$ and $\lambda>0, \lambda B$ denotes the ball concentric with $B$ whose radius is $\lambda$ times as long. For a given weight function $\omega$ and a measurable set $E$, we also denote the Lebesgue measure of $E$ by $|E|$ and set the weighted measure $\omega(E)=\int_{E} \omega(x) d x$.

Let $1 \leq p<\infty$, let $\varphi$ be a positive measurable function on $\mathbb{R}^{n} \times(0, \infty)$, and let $\omega$ be a nonnegative measurable function on $\mathbb{R}^{n}$. Following [14], we denote by $M_{\varphi}^{p}(\omega)$ the generalized weighted Morrey space and the space of all functions $f \in$ $L_{\mathrm{loc}}^{p}(\omega)$ with finite norm

$$
\|f\|_{M_{\varphi}^{p}(w)}=\sup _{x \in \mathbb{R}^{n}, r>0} \frac{1}{\varphi(x, r)}\left(\frac{1}{w(B(x, r))}\|f\|_{L^{p}(\omega, B(x, r))}^{p}\right)^{1 / p},
$$

where

$$
\|f\|_{L^{p}(\omega, B(x, r))}=\int_{B(x, r)}|f(y)|^{p} w(y) d y .
$$

Furthermore, by $W M_{\varphi}^{p}(\omega)$ we denote the weak generalized weighted Morrey space of all functions $f \in W M_{\varphi}^{p}(\omega)$ for which

$$
\|f\|_{W M_{\varphi}^{p}(w)}=\sup _{x \in \mathbb{R}^{n}, r>0} \frac{1}{\varphi(x, r)}\left(\frac{1}{w(B(x, r))}\|f\|_{W L^{p}(\omega, B(x, r))}^{p}\right)^{1 / p},
$$

where

$$
\|f\|_{W L^{p}(\omega, B(x, r))}=\sup _{t>0} t(\omega(\{y \in B(x, r):|f(y)|>t\}))^{1 / p} .
$$

(1) If $\omega=1$ and $\varphi(x, r)=r^{(\lambda-n) / p}$ with $0<\lambda<n$, then $M_{\varphi}^{p}(\omega)=L^{p, \lambda}$ is the classical Morrey space. 
(2) If $\varphi(x, r)=\omega(B(x, r))^{(\kappa-1) / p}$, then $M_{\varphi}^{p}(\omega)=L^{p, \kappa}(\omega)$ is the weighted Morrey space.

(3) If $\varphi(x, r)=\nu(B(x, r))^{\kappa / p} \omega(B(x, r))^{-1 / p}$, then $M_{\varphi}^{p}(\omega)=$ $L^{p, \kappa}(\nu, \omega)$ is the two weighted Morrey space.

(4) If $\omega=1$, then $M_{\varphi}^{p}(\omega)=M_{\varphi}^{p}$ is the generalized Morrey space.

(5) If $\varphi(x, r)=\omega(B(x, r))^{-1 / p}$, then $M_{\varphi}^{p}(\omega)=L^{p}(\omega)$.

A weight $\omega$ is said to belong to $A_{p}$, for $1<p<\infty$, if there exists a constant $C$ such that, for every ball $B \subset \mathbb{R}^{n}$,

$$
\left(\frac{1}{|B|} \int_{B} \omega(x) d x\right)\left(\frac{1}{|B|} \int_{B} \omega(x)^{1-p^{\prime}} d x\right)^{p-1} \leq C,
$$

where $s^{\prime}$ is the dual of $s$ such that $1 / s+1 / s^{\prime}=1$. The class $A_{1}$ is defined by replacing the above inequality with

$\frac{1}{|B|} \int_{B} w(y) d y \leq C \cdot \underset{x \in B}{\text { ess inf }} w(x) \quad$ for every ball $B \subset \mathbb{R}^{n}$.

A weight $\omega$ is said to belong to $A_{\infty}$ if there are positive numbers $C$ and $0<\delta<1$ so that

$$
\frac{\omega(E)}{\omega(B)} \leq C\left(\frac{|E|}{|B|}\right)^{\delta}
$$

for all balls $B$ and all measurable $E \subset B$. It is well known that

$$
A_{\infty}=\bigcup_{1 \leq p<\infty} A_{p}
$$

By (26), we have

$$
\left(\int_{B} \omega(x) d x\right)\left(\int_{B} \omega(x)^{1-p^{\prime}} d x\right)^{p-1} \leq C|B|^{p}
$$

for $1<p<\infty$. Note that

$$
(\underset{x \in E}{\operatorname{ess} \inf } f(x))^{-1}=\underset{x \in E}{\operatorname{ess} \sup _{x \in}} \frac{1}{f(x)}
$$

is true for any real-valued nonnegative function $f$ and measurable on $E$ (see [18], p.143) and, by (27), we get

$$
\begin{aligned}
\left\|\omega^{-1}\right\|_{L^{\infty}(B)} & =\underset{x \in B}{\operatorname{ess} \sup } \frac{1}{w(x)}=\frac{1}{\operatorname{ess~inf}_{x \in B} w(x)} \\
& \leq C|B| \omega(B)^{-1} .
\end{aligned}
$$

The classical $A_{p}$ weight theory was first introduced by Muckenhoupt in the study of weighted $L^{p}$ boundedness of Hardy-Littlewood maximal function in [19].

Lemma 7 (see $[19,20]$ ). Suppose that $\omega \in A_{p}$ and the following statements hold.

(i) For any $1 \leq p<\infty$, there are positive numbers $C$ and $0<\delta<1$ such that

$$
\begin{aligned}
& \omega(2 B) \leq C 2^{n p} \omega(B), \\
& \omega(2 B) \geq C 2^{n \delta} \omega(B) ;
\end{aligned}
$$

(ii) $A_{p_{1}} \subset A_{p_{2}}$ for any $1 \leq p_{1}<p_{2} \leq \infty$;

(iii) for any $1<p<\infty$, one has $\omega^{1-p^{\prime}} \in A_{p^{\prime}}$.

Now, let us recall the definition of multiple weights. For $m$ exponents $p_{1}, \ldots, p_{m}$, we write $\vec{p}=\left(p_{1}, \ldots, p_{m}\right)$. Let $p_{1}, \ldots, p_{m} \in[1, \infty)$, and let $p \in(0, \infty)$ with $1 / p=$ $\sum_{k=1}^{m} 1 / p_{k}, \vec{\omega}=\left(\omega_{1}, \ldots, \omega_{m}\right)$. Given $\vec{\omega}=\left(\omega_{1}, \ldots, \omega_{m}\right)$, set $\nu_{\vec{\omega}}=\prod_{i=1}^{m} \omega_{i}^{p / p_{i}}$. We say that $\vec{\omega}$ satisfies the $A_{\vec{p}}$ condition if it satisfies

$$
\sup _{B}\left(\frac{1}{|B|} \int_{B} \nu_{\vec{\omega}}(x) d x\right)^{1 / p} \prod_{i=1}^{m}\left(\frac{1}{|B|} \int_{B} \omega_{i}(x)^{1-p_{i}^{\prime}}(x) d x\right)^{1 / p_{i}^{\prime}}
$$

$<\infty$

When $p_{i}=1,(1 /|B|) \int_{B} \omega_{i}(x)^{1-p_{i}^{\prime}}(x) d x$ is understood as $\left(\inf _{x \in B} \omega_{i}(x)\right)^{-1}$.

Lemma 8 (see [5]). Let $p_{1}, \ldots, p_{m} \in[1, \infty)$, and let $1 / p=$ $\sum_{k=1}^{m} 1 / p_{k}$. Then, $\vec{\omega}=\left(\omega_{1}, \ldots, \omega_{m}\right) \in A_{\vec{p}}$ if and only if

$$
\nu_{\vec{\omega}} \in A_{p m}, \quad \omega_{i}^{1-p_{i}^{\prime}} \in A_{m p_{i}^{\prime}} \quad \text { for } i=1, \ldots, m,
$$

where $\nu_{\vec{\omega}}=\prod_{i=1}^{m} \omega_{i}^{p / p_{i}}$ and the condition $\omega_{i}^{1-p_{i}^{\prime}} \in A_{m p_{i}^{\prime}}$ in the case $p_{i}=1$ is understood as $\omega_{i}^{1 / m} \in A_{1}$.

Lemma 9 (see [6]). Let $p_{1}, \ldots, p_{m} \in[1, \infty)$, and let $p \in$ $(0, \infty)$ with $1 / p=\sum_{k=1}^{m} 1 / p_{k}$. Assume that $\omega_{1}, \ldots, \omega_{m} \in A_{\infty}$ and $\nu_{\vec{\omega}}=\prod_{i=1}^{m} \omega_{i}^{p / p_{i}}$. Then, for any ball $B$, there exists $a$ constant $C>0$ such that

$$
\prod_{i=1}^{m}\left(\int_{B} \omega_{i}(x) d x\right)^{p / p_{i}} \leq C \int_{B} \nu_{\vec{\omega}}(x) d x
$$

Let us recall the definition and some properties of $B M O$. Following [21], a locally integrable function $b$ is said to be in $B M O$ if

$$
\sup _{B \subset \mathbb{R}^{n}} \frac{1}{|B|} \int_{B}\left|b(x)-b_{B}\right| d x=\|b\|_{*}<\infty
$$

where $b_{B}=|B|^{-1} \int_{B} b(y) d y$.

Lemma 10 (see [22]). Suppose that $\omega \in A_{\infty}$ and $b \in B M O$. Then, for any $p \geq 1$, one has

$$
\left(\frac{1}{\omega(B)} \int_{B}\left|b(x)-b_{B}\right|^{p} \omega(x) d x\right)^{1 / p} \leq C\|b\|_{*} .
$$

Lemma 11 (see [23]). Let $b \in B M O, 1 \leq p<\infty$, and $r_{1}, r_{2}>$ 0 . Then,

$$
\begin{aligned}
& \left(\frac{1}{\left|B\left(x_{0}, r_{1}\right)\right|} \int_{B\left(x_{0}, r_{1}\right)}\left|b(y)-b_{B\left(x_{0}, r_{2}\right)}\right|^{p} d y\right)^{1 / p} \\
& \leq C\|b\|_{*}\left(1+\left|\ln \frac{r_{1}}{r_{2}}\right|\right)
\end{aligned}
$$

where $C>0$ is independent of $f, x_{0}, r_{1}$, and $r_{2}$. 
By Lemmas 10 and 11, it is easy to prove the following results.

Lemma 12. Suppose that $\omega \in A_{\infty}$ and $b \in B M O$. Then, for any $1 \leq p<\infty$ and $r_{1}, r_{2}>0$, one has

$$
\begin{aligned}
& \left(\frac{1}{\omega\left(B\left(x_{0}, r_{1}\right)\right)} \int_{B\left(x_{0}, r_{1}\right)}\left|b(x)-b_{B\left(x_{0}, r_{2}\right)}\right|^{p} \omega(x) d x\right)^{1 / p} \\
& \leq C\|b\|_{*}\left(1+\left|\ln \frac{r_{1}}{r_{2}}\right|\right) .
\end{aligned}
$$

\section{Proof of Theorem 2}

We first prove the following conclusions.

Theorem 13. Let $m \geq 2$, let $T$ be an $m$-linear CalderónZygmund operator, and let $\vec{\omega}=\left(\omega_{1}, \ldots, \omega_{m}\right)$ satisfy the $A_{\vec{p}}$ condition with $\omega_{1}, \ldots, \omega_{m} \in A_{\infty}$. If $p_{1}, \ldots, p_{m} \in(1, \infty)$ and $p \in(0, \infty)$ with $1 / p=\sum_{i=1}^{m} 1 / p_{i}$, then, for any $x_{0} \in \mathbb{R}^{n}$,

$$
\begin{aligned}
\|T(\vec{f})\|_{L^{p}\left(v_{\vec{\omega}}, B\left(x_{0}, s\right)\right)} \\
\leq C \prod_{i=1}^{m} \omega_{i}\left(B\left(x_{0}, s\right)\right)^{1 / p_{i}} \\
\quad \times \int_{2 s}^{\infty}\left(\prod_{i=1}^{m}\left\|f_{i}\right\|_{L^{p_{i}\left(\omega_{i}, B\left(x_{0}, r\right)\right)}} \omega_{i}\left(B\left(x_{0}, r\right)\right)^{-1 / p_{i}}\right) \frac{d r}{r} .
\end{aligned}
$$

If $p_{1}, \ldots, p_{m} \in[1, \infty), \min \left\{p_{1}, \ldots, p_{m}\right\}=1$, and $p \in(0, \infty)$ with $1 / p=\sum_{i=1}^{m} 1 / p_{i}$, then, for any $x_{0} \in \mathbb{R}^{n}$,

$$
\begin{aligned}
& \|T(\vec{f})\|_{W L^{p}\left(v_{\vec{\omega}}, B\left(x_{0}, s\right)\right)} \\
& \leq C \prod_{i=1}^{m} \omega_{i}\left(B\left(x_{0}, s\right)\right)^{1 / p_{i}} \\
& \quad \times \int_{2 s}^{\infty}\left(\prod_{i=1}^{m}\left\|f_{i}\right\|_{L^{p_{i}}\left(\omega_{i}, B\left(x_{0}, r\right)\right)} \omega_{i}\left(B\left(x_{0}, r\right)\right)^{-1 / p_{i}}\right) \frac{d r}{r} .
\end{aligned}
$$

Proof. We represent $f_{i}$ as $f_{i}=f_{i}^{0}+f_{i}^{\infty}$, where $f_{i}^{0}=f_{i} \chi_{B\left(x_{0}, 2 s\right)}$, $i=1, \ldots, m$, and $\chi_{B\left(x_{0}, 2 s\right)}$ denotes the characteristic function of $B\left(x_{0}, 2 s\right)$. Then,

$$
\begin{aligned}
\prod_{i=1}^{m} f_{i}\left(y_{i}\right) & =\prod_{i=1}^{m}\left(f_{i}^{0}\left(y_{i}\right)+f_{i}^{\infty}\left(y_{i}\right)\right) \\
& =\sum_{\alpha_{1}, \ldots, \alpha_{m} \in\{0, \infty\}} f_{1}^{\alpha_{1}}\left(y_{1}\right) \cdots f_{m}^{\alpha_{m}}\left(y_{m}\right) \\
& =\prod_{i=1}^{m} f_{i}^{0}\left(y_{i}\right)+\Sigma^{\prime} f_{1}^{\alpha_{1}}\left(y_{1}\right) \cdots f_{m}^{\alpha_{m}}\left(y_{m}\right),
\end{aligned}
$$

where each term of $\Sigma^{\prime}$ contains at least one $\alpha_{i} \neq 0$. Since $T$ is an $m$-linear operator, then

$$
\begin{aligned}
\|T(\vec{f})\|_{L^{p}\left(v_{\vec{\omega}}, B\left(x_{0}, s\right)\right)} \leq & C\left\|T\left(f_{1}^{0}, \ldots, f_{m}^{0}\right)\right\|_{L^{p}\left(v_{\vec{\omega}}, B\left(x_{0}, s\right)\right)} \\
& +C \Sigma^{\prime}\left\|T\left(f_{1}^{\alpha_{1}}, \ldots, f_{m}^{\alpha_{m}}\right)\right\|_{L^{p}\left(v_{\vec{\omega}}, B\left(x_{0}, s\right)\right)} \\
= & I^{0, \ldots, 0}+\Sigma^{\prime} I^{\alpha_{1}, \ldots, \alpha_{m}}, \\
\|T(\vec{f})\|_{W L^{p}\left(v_{\vec{\omega}}, B\left(x_{0}, s\right)\right)} \leq & C\left\|T\left(f_{1}^{0}, \ldots, f_{m}^{0}\right)\right\|_{W L^{p}\left(v_{\vec{\omega}}, B\left(x_{0}, s\right)\right)} \\
& +C \Sigma^{\prime}\left\|T\left(f_{1}^{\alpha_{1}}, \ldots, f_{m}^{\alpha_{m}}\right)\right\|_{W L^{p}\left(v_{\vec{\omega}}, B\left(x_{0}, s\right)\right)} \\
= & J^{0, \ldots, 0}+\Sigma^{\prime} J^{\alpha_{1}, \ldots, \alpha_{m}} .
\end{aligned}
$$

Then, by Theorem 1 , if $1<p_{i}<\infty, i=1, \ldots, m$, we get

$$
I^{0, \ldots, 0} \leq C \prod_{i=1}^{m}\left\|f_{i}\right\|_{L^{p_{i}\left(\omega_{i}, B\left(x_{0}, 2 s\right)\right)}}
$$

If $1 \leq p_{i}<\infty, \min \left\{p_{1}, \ldots, p_{m}\right\}=1$, then

$$
J^{0, \ldots, 0} \leq C \prod_{i=1}^{m}\left\|f_{i}\right\|_{L^{p_{i}}\left(\omega_{i}, B\left(x_{0}, 2 s\right)\right)}
$$

Applying Hölder's inequality, for $1 \leq p_{i}<\infty, i=1, \ldots, m$, we have

$$
\left|B\left(x_{0}, 2 s\right)\right| \leq \omega_{i}\left(B\left(x_{0}, 2 s\right)\right)^{1 / p_{i}}\left\|\omega_{i}^{-1 / p_{i}}\right\|_{L^{p_{i}^{\prime}\left(B\left(x_{0}, 2 s\right)\right)}} .
$$

Thus, for $1 \leq p_{i}<\infty$,

$$
\begin{aligned}
& \prod_{i=1}^{m}\left\|f_{i}\right\|_{L^{p_{i}}\left(\omega_{i}, B\left(x_{0}, 2 s\right)\right)} \\
& \leq C \prod_{i=1}^{m}\left\|f_{i}\right\|_{L^{p_{i}}\left(\omega_{i}, B\left(x_{0}, 2 s\right)\right)} \cdot\left|B\left(x_{0}, 2 s\right)\right|^{m} \int_{2 s}^{\infty} \frac{d r}{r^{m n+1}} \\
& \leq C \prod_{i=1}^{m}\left(\omega_{i}\left(B\left(x_{0}, 2 s\right)\right)^{1 / p_{i}}\left\|f_{i}\right\|_{L^{p_{i}}\left(\omega_{i}, B\left(x_{0}, 2 s\right)\right)}\right. \\
& \left.\times\left\|\omega_{i}^{-1 / p_{i}}\right\|_{L^{p_{i}^{\prime}\left(B\left(x_{0}, 2 s\right)\right)}}\right) \int_{2 s}^{\infty} \frac{d r}{r^{m n+1}} \\
& \leq C \prod_{i=1}^{m} \omega_{i}\left(B\left(x_{0}, 2 s\right)\right)^{1 / p_{i}} \\
& \cdot \int_{2 s}^{\infty}\left(\prod_{i=1}^{m}\left\|f_{i}\right\|_{L^{p_{i}\left(\omega_{i}, B\left(x_{0}, r\right)\right)}}\left\|\omega_{i}^{-1 / p_{i}}\right\|_{L^{p_{i}^{\prime}\left(B\left(x_{0}, r\right)\right)}}\right) \\
& \times \frac{d r}{r^{m n+1}} .
\end{aligned}
$$


From (35) and Lemma 9, we get

$$
\begin{aligned}
& \prod_{i=1}^{m}\left\|\omega_{i}^{-1 / p_{i}}\right\|_{L^{p_{i}^{\prime}}\left(B\left(x_{0}, r\right)\right)} \\
& \quad \leq C\left|B\left(x_{0}, r\right)\right|^{1 / p+\sum_{i=1}^{m}\left(1 / p_{i}^{\prime}\right)}\left(\int_{B\left(x_{0}, r\right)} \nu_{\vec{\omega}}(x) d x\right)^{-1 / p} \\
& \quad \leq C\left|B\left(x_{0}, r\right)\right|^{m} \prod_{i=1}^{m} \omega_{i}\left(B\left(x_{0}, r\right)\right)^{-1 / p_{i}} .
\end{aligned}
$$

Then, for $1 \leq p_{i}<\infty, i=1, \ldots, m$,

$$
\begin{aligned}
\prod_{i=1}^{m}\left\|f_{i}\right\|_{L^{p_{i}}\left(\omega_{i}, B\left(x_{0}, 2 s\right)\right)} & \\
\leq & C \prod_{i=1}^{m} \omega_{i}\left(B\left(x_{0}, s\right)\right)^{1 / p_{i}} \\
& \cdot \int_{2 s}^{\infty}\left(\prod_{i=1}^{m}\left\|f_{i}\right\|_{L^{p_{i}}\left(\omega_{i}, B\left(x_{0}, r\right)\right)} \omega_{i}\left(B\left(x_{0}, r\right)\right)^{-1 / p_{i}}\right) \frac{d r}{r} .
\end{aligned}
$$

This gives that $I^{0, \ldots, 0}$ and $J^{0, \ldots, 0}$ are majored by

$$
\begin{aligned}
C \prod_{i=1}^{m} \omega_{i}\left(B\left(x_{0}, s\right)\right)^{1 / p_{i}} \\
\quad \cdot \int_{2 s}^{\infty}\left(\prod_{i=1}^{m}\left\|f_{i}\right\|_{L^{p_{i}\left(\omega_{i}, B\left(x_{0}, r\right)\right)}} \omega_{i}\left(B\left(x_{0}, r\right)\right)^{-1 / p_{i}}\right) \frac{d r}{r} .
\end{aligned}
$$

For the other term, let us first consider the case when $\alpha_{1}=$ $\cdots=\alpha_{m}=\infty$. By the size condition (1), for any $x \in B\left(x_{0}, s\right)$, we obtain

$$
\begin{aligned}
& \left|T\left(f_{1}^{\infty}, \ldots, f_{m}^{\infty}\right)(x)\right| \\
& \quad \leq C \int_{\left(\mathbb{R}^{n} \backslash B\left(x_{0}, 2 s\right)\right)^{m}} \frac{\left|f_{1}\left(y_{1}\right) \cdots f_{m}\left(y_{m}\right)\right|}{\left(\left|x-y_{1}\right|+\cdots+\left|x-y_{m}\right|\right)^{m n}} d y_{1} \cdots d y_{m} \\
& \quad \leq C \sum_{j=1}^{\infty} \int_{\left(B\left(x_{0}, 2^{j+1} s\right) \backslash B\left(x_{0}, 2^{j} s\right)\right)^{m}}\left(\left|f_{1}\left(y_{1}\right) \cdots f_{m}\left(y_{m}\right)\right|\right) \\
& \quad \leq C \sum_{j=1}^{\infty} \prod_{i=1}^{m} \int_{B\left(x_{0}, 2^{j+1} s\right) \backslash B\left(x_{0}, 2^{j} s\right)} \frac{\left|f_{i}\left(y_{i}\right)\right|}{\left|x-y_{i}\right|^{n}} d y_{i} \\
& \quad \leq C \sum_{j=1}^{\infty} \prod_{i=1}^{m}\left(\left(2^{j+1} s\right)^{-n} \int_{B\left(x_{0}, 2^{j+1} s\right)}\left|f_{i}\left(y_{i}\right)\right| d y_{i}\right) .
\end{aligned}
$$

Applying Hölder's inequality, it can be found that $\sup _{x \in B\left(x_{0}, s\right)}\left|T\left(f_{1}^{\infty}, \ldots, f_{m}^{\infty}\right)(x)\right|$ is less than

$$
C \sum_{j=1}^{\infty} \prod_{i=1}^{m}\left(\left(2^{j+1} s\right)^{-n}\left\|f_{i}\right\|_{L^{p_{i}}\left(\omega_{i}, B\left(x_{0}, 2^{j+1} s\right)\right)}\left\|\omega_{i}^{-1 / p_{i}}\right\|_{L^{p^{\prime}}\left(B\left(x_{0}, 2^{j+1} s\right)\right)}\right) \text {. }
$$

Hence,

$$
\begin{aligned}
& \sup _{x \in B\left(x_{0}, s\right)}\left|T\left(f_{1}^{\infty}, \ldots, f_{m}^{\infty}\right)(x)\right| \\
& \leq C \sum_{j=1}^{\infty} \int_{2^{j+1} s}^{2^{j+2} s}\left(2^{j+2} s\right)^{-n m-1}
\end{aligned}
$$

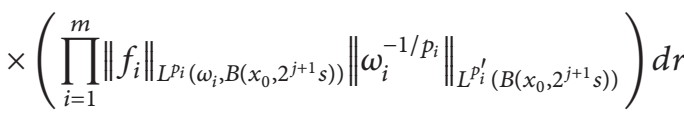

$$
\begin{aligned}
& \leq C \sum_{j=1}^{\infty} \int_{2^{j+1} s}^{2^{j+2} s}\left(\prod_{i=1}^{m}\left\|f_{i}\right\|_{L^{p_{i}}\left(\omega_{i}, B\left(x_{0}, 2^{j+1} s\right)\right)}\right. \\
& \left.\times\left\|\omega_{i}^{-1 / p_{i}}\right\|_{L^{p_{i}^{\prime}\left(B\left(x_{0}, 2^{j+1} s\right)\right)}}\right) \frac{d r}{r^{m n+1}} \\
& \leq C \int_{2 s}^{\infty}\left(\prod_{i=1}^{m}\left\|f_{i}\right\|_{L^{p_{i}}\left(\omega_{i}, B\left(x_{0}, 2^{j+1} s\right)\right)}\left\|\omega_{i}^{-1 / p_{i}}\right\|_{L^{p_{i}^{\prime}}\left(B\left(x_{0}, 2^{j+1} s\right)\right)}\right) \frac{d r}{r^{m n+1}} .
\end{aligned}
$$

Substituting (50) into above, we obtain

$$
\begin{aligned}
& \sup _{x \in B\left(x_{0}, s\right)}\left|T\left(f_{1}^{\infty}, \ldots, f_{m}^{\infty}\right)(x)\right| \\
& \quad \leq C \int_{2 s}^{\infty}\left(\prod_{i=1}^{m}\left\|f_{i}\right\|_{L^{p_{i}}\left(\omega_{i}, B\left(x_{0}, r\right)\right)} \omega_{i}\left(B\left(x_{0}, r\right)\right)^{-1 / p_{i}}\right) \frac{d r}{r} .
\end{aligned}
$$

Using Hölder's inequality,

$$
\left(\int_{B\left(x_{0}, s\right)} \nu_{\vec{\omega}}(x) d x\right)^{1 / p} \leq C \prod_{i=1}^{m} \omega_{i}\left(B\left(x_{0}, s\right)\right)^{1 / p_{i}} .
$$

From (56) and (57), we know that $I^{\infty, \ldots, \infty}$ and $J^{\infty, \ldots, \infty}$ are not greater than (52) for $1 \leq p_{i}<\infty, i=1, \ldots, m$.

Now, we consider the case where exactly $\tau$ of the $\alpha_{i}$ are $\infty$ for some $1 \leq \tau<m$. We only give the arguments for one of the cases. The rest are similar and can easily be obtained from the arguments below by permuting the indices. Applying the size condition again, we deduce that, for any $x \in B\left(x_{0}, s\right)$,

$$
\begin{aligned}
& \left|T\left(f_{1}^{\infty}, \ldots, f_{\tau}^{\infty}, f_{\tau+1}^{0}, \ldots, f_{m}^{0}\right)(x)\right| \\
& \leq C \int_{\left(\mathbb{R}^{n} \backslash B\left(x_{0}, 2 s\right)\right)^{\tau}} \int_{\left(B\left(x_{0}, 2 s\right)\right)^{m-\tau}}\left(\left|f_{1}\left(y_{1}\right) \cdots f_{m}\left(y_{m}\right)\right|\right) \\
& \quad \times\left(\left(\left|x-y_{1}\right|+\cdots\right.\right. \\
& \left.\left.\quad+\left|x-y_{m}\right|\right)^{m n}\right)^{-1} d y_{1} \cdots d y_{m}
\end{aligned}
$$


Journal of Function Spaces

7

$$
\begin{aligned}
\leq & C \prod_{i=\tau+1}^{m} \int_{B\left(x_{0}, 2 s\right)}\left|f_{i}\left(y_{i}\right)\right| d y_{i} \\
\times & \sum_{j=1}^{\infty} \frac{1}{\left|B\left(x_{0}, 2^{j+1} s\right)\right|^{m}} \\
\times & \int_{\left(B\left(x_{0}, 2^{j+1} s\right) \backslash B\left(x_{0}, 2^{j} s\right)\right)^{\tau}}\left|f_{1}\left(y_{1}\right) \cdots f_{\tau}\left(y_{\tau}\right)\right| d y_{1} \cdots d y_{\tau} \\
\leq & C \prod_{i=\tau+1}^{m} \int_{B\left(x_{0}, 2 s\right)}\left|f_{i}\left(y_{i}\right)\right| d y_{i} \\
& \cdot \sum_{j=1}^{\infty} \frac{1}{\left|B\left(x_{0}, 2^{j+1} s\right)\right|^{m}} \prod_{i=1}^{\tau} \int_{B\left(x_{0}, 2^{j+1} s\right) \backslash B\left(x_{0}, 2^{j} s\right)}\left|f_{i}\left(y_{i}\right)\right| d y_{i} \\
\leq & C \sum_{j=1}^{\infty} \prod_{i=1}^{m} \frac{1}{\left|B\left(x_{0}, 2^{j+1} s\right)\right|} \int_{B\left(x_{0}, 2^{j+1} s\right)}\left|f_{i}\left(y_{i}\right)\right| d y_{i} .
\end{aligned}
$$

Similar to the estimates for $I^{\infty, \ldots, \infty}$, we get

$$
\begin{aligned}
& \sup _{x \in B\left(x_{0}, s\right)}\left|T\left(f_{1}^{\infty}, \ldots, f_{\tau}^{\infty}, f_{\tau+1}^{0}, \ldots, f_{m}^{0}\right)(x)\right| \\
& \leq C \int_{2 s}^{\infty}\left(\prod_{i=1}^{m}\left\|f_{i}\right\|_{L^{p_{i}}\left(\omega_{i}, B\left(x_{0}, r\right)\right)} \omega_{i}\left(B\left(x_{0}, r\right)\right)^{-1 / p_{i}}\right) \frac{d r}{r} .
\end{aligned}
$$

Then, $I^{\infty, \ldots, \infty, 0, \ldots, 0}$ and $J^{\infty, \ldots, \infty, 0, \ldots, 0}$ are all less than

$$
\begin{aligned}
C \prod_{i=1}^{m} \omega_{i}\left(B\left(x_{0}, s\right)\right)^{1 / p_{i}} \\
\quad \cdot \int_{2 s}^{\infty}\left(\prod_{i=1}^{m}\left\|f_{i}\right\|_{L^{p_{i}\left(\omega_{i}, B\left(x_{0}, r\right)\right)}} \omega_{i}\left(B\left(x_{0}, r\right)\right)^{-1 / p_{i}}\right) \frac{d r}{r} .
\end{aligned}
$$

Combining the above estimates, we complete the proof of Theorem 13.

Now, we can give the proof of Theorem 2. From the definition of generalized Murrey space, the norm of $T(\vec{f})$ on $M_{\varphi_{2}}^{p}\left(\nu_{\vec{\omega}}\right)$ equals

$$
\begin{aligned}
\sup _{x \in R^{n}, r>0}\left(\prod_{i=1}^{m} \varphi_{2 i}(x, s)\right)^{-1} & \left(\frac{1}{v_{\vec{\omega}}(B(x, s))}\right. \\
& \left.\times \int_{B(x, s)}|T(\vec{f})(y)|^{p} \nu_{\vec{\omega}}(y) d y\right)^{1 / p} .
\end{aligned}
$$

By (37), we get

$$
\left(\int_{B(x, s)} \nu_{\vec{\omega}}(x) d x\right)^{-1 / p} \leq C \prod_{i=1}^{m}\left(\int_{B(x, s)} \omega_{i}(x) d x\right)^{-1 / p_{i}} .
$$

Combining (42) and (62),

$$
\begin{aligned}
& \left(\frac{1}{v_{\vec{\omega}}(B(x, s))} \int_{B(x, s)}|T(\vec{f})(y)|^{p} v_{\vec{\omega}}(y) d y\right)^{1 / p} \\
& \quad \leq C \int_{s}^{\infty}\left(\prod_{i=1}^{m}\left\|f_{i}\right\|_{L^{p_{i}\left(\omega_{i}, B(x, r)\right)}} \omega_{i}(B(x, r))^{-1 / p_{i}}\right) \frac{d r}{r} .
\end{aligned}
$$

Since $f_{i} \in M_{\varphi_{1 i}}^{p_{i}}\left(\omega_{i}\right)$, then, by (31) and the fact that $\left\|f_{i}\right\|_{L^{p_{i}(B(x, r))}}$ are all nondecreasing function of $r$, we get

$$
\begin{gathered}
\frac{\prod_{i=1}^{m}\left\|f_{i}\right\|_{L^{p_{i}}\left(\omega_{i}, B(x, r)\right)}}{{\text { uss } \inf _{r<t<\infty}}_{\prod_{i=1}^{m} \varphi_{1 i}(x, t) \omega_{i}(B(x, t))^{1 / p_{i}}}} \\
\leq \operatorname{ess} \sup _{0<r<t<\infty} \frac{\prod_{i=1}^{m}\left\|f_{i}\right\|_{L^{p_{i}}\left(\omega_{i}, B(x, r)\right)}}{\prod_{i=1}^{m} \varphi_{1 i}(x, t) \omega_{i}(B(x, t))^{1 / p_{i}}} \\
\leq \operatorname{ess} \sup _{0<t<\infty} \frac{\prod_{i=1}^{m}\left\|f_{i}\right\|_{L^{p_{i}}\left(\omega_{i}, B(x, t)\right)}}{\prod_{i=1}^{m} \varphi_{1 i}(x, t) \omega_{i}(B(x, t))^{1 / p_{i}}} \\
\leq C \prod_{i=1}^{m}\left\|f_{i}\right\|_{M_{\varphi_{1 i}}^{p_{i}}\left(\omega_{i}\right)} .
\end{gathered}
$$

Then,

$$
\begin{aligned}
& \int_{s}^{\infty}\left(\prod_{i=1}^{m}\left\|f_{i}\right\|_{L^{p_{i}}\left(\omega_{i}, B(x, r)\right)} \omega_{i}(B(x, r))^{-1 / p_{i}}\right) \frac{d r}{r} \\
& =\int_{s}^{\infty} \frac{\prod_{i=1}^{m}\left\|f_{i}\right\|_{L^{p_{i}}\left(\omega_{i}, B(x, r)\right)}}{\operatorname{ess}_{\inf } \prod_{r<t<\infty}^{m} \prod_{i=1}^{m} \varphi_{1 i}(x, t) \omega_{i}(B(x, t))^{1 / p_{i}}} \\
& \times \frac{\operatorname{ess~inf}_{r<t<\infty} \prod_{i=1}^{m} \varphi_{1 i}(x, t) \omega_{i}(B(x, t))^{1 / p_{i}}}{\prod_{i=1}^{m} \omega_{i}(B(x, t))^{1 / p_{i}}} \frac{d r}{r} \\
& \leq C \prod_{i=1}^{m}\left\|f_{i}\right\|_{M_{\varphi_{1 i}}^{p_{i}}\left(\omega_{i}\right)} \\
& \times \int_{s}^{\infty} \frac{\operatorname{ess~inf}_{r<t<\infty} \prod_{i=1}^{m} \varphi_{1 i}(x, t) \omega_{i}(B(x, t))^{1 / p_{i}}}{\prod_{i=1}^{n} \omega_{i}(B(x, t))^{1 / p_{i}}} \frac{d r}{r} .
\end{aligned}
$$

By condition (8), we get

$$
\begin{aligned}
& \int_{s}^{\infty}\left(\prod_{i=1}^{m}\left\|f_{i}\right\|_{L^{p_{i}}\left(\omega_{i}, B(x, r)\right)} \omega_{i}(B(x, r))^{1 / p_{i}}\right) \frac{d r}{r} \\
& \quad \leq C \prod_{i=1}^{m}\left\|f_{i}\right\|_{M_{\varphi_{1 i}}^{p_{i}}\left(\omega_{i}\right)} \prod_{i=1}^{m} \varphi_{2 i}(x, s) .
\end{aligned}
$$

Combining (61), (63), and (66), the proof of the first part of Theorem 2 is completed.

Similarly, the norm of $T(\vec{f})$ on $W M_{\varphi_{2}}^{p}\left(\nu_{\vec{\omega}}\right)$ equals

$$
\begin{aligned}
\sup _{x \in R^{n}, r>0} & \left(\prod_{i=1}^{m} \varphi_{2 i}(x, s)\right)^{-1} \\
& \times\left(\frac{1}{v_{\vec{\omega}}(B(x, s))}\|T(\vec{f})\|_{W L^{p}\left(v_{\vec{\omega}}, B(x, s)\right)}^{p}\right)^{1 / p} .
\end{aligned}
$$


Combining (43) and (62),

$$
\begin{aligned}
& \left(\frac{1}{\nu_{\vec{\omega}}(B(x, s))}\|T(\vec{f})\|_{W L^{p}\left(v_{\vec{\omega}}, B(x, s)\right)}^{p}\right)^{1 / p} \\
& \quad \leq C \int_{s}^{\infty}\left(\prod_{i=1}^{m}\left\|f_{i}\right\|_{L^{p_{i}\left(\omega_{i}, B(x, r)\right)}} \omega_{i}(B(x, r))^{-1 / p_{i}}\right) \frac{d r}{r}
\end{aligned}
$$

Substituting (66) into (68),

$$
\begin{gathered}
\left(\frac{1}{v_{\vec{\omega}}(B(x, s))}\|T(\vec{f})\|_{W L^{p}\left(v_{\vec{\omega}}, B(x, s)\right)}^{p}\right)^{1 / p} \\
\leq C \varphi_{2}(x, s) \prod_{i=1}^{m}\left\|f_{i}\right\|_{M_{\varphi_{1 i}}^{p_{i}}\left(\omega_{i}\right)}
\end{gathered}
$$

Then,

$$
\|T(\vec{f})\|_{W M_{\varphi_{2}}^{p}\left(v_{\vec{\omega}}\right)} \leq C \prod_{i=1}^{m}\left\|f_{i}\right\|_{M_{\varphi_{1 i}}^{p_{i}}\left(\omega_{i}\right)}
$$

This completes the proof of the second part of Theorem 2 .

\section{Proof of Theorem 3}

Theorem 14. Let $m \geq 2$, let $T$ be an m-linear CalderónZygmund operator, and let $\vec{\omega}=\left(\omega_{1}, \ldots, \omega_{m}\right)$ satisfy the $A_{\vec{p}}$ condition with $\omega_{1}, \ldots, \omega_{m} \in A_{\infty}$. If $p_{1}, \ldots, p_{m} \in(1, \infty)$ and $p \in(0, \infty)$ with $1 / p=\sum_{i=1}^{m} 1 / p_{i}$, then, for any $x_{0} \in \mathbb{R}^{n}$,

$$
\begin{aligned}
& \left\|T_{\Sigma b}(\vec{f})\right\|_{L^{p}\left(v_{\vec{\omega}}, B\left(x_{0}, s\right)\right)} \\
& \leq C \prod_{i=1}^{m}\left(\left\|b_{i}\right\|_{*} \omega_{i}\left(B\left(x_{0}, s\right)\right)^{1 / p_{i}}\right) \\
& \quad \times \int_{2 s}^{\infty}\left(1+\ln \frac{r}{s}\right)^{m} \\
& \quad \times\left(\prod_{i=1}^{m}\left\|f_{i}\right\|_{L^{p_{i}\left(\omega_{i}, B\left(x_{0}, r\right)\right)}} \omega_{i}\left(B\left(x_{0}, r\right)\right)^{-1 / p_{i}}\right) \frac{d r}{r},
\end{aligned}
$$

$$
\begin{aligned}
& \left\|T_{\Pi b}(\vec{f})\right\|_{L^{p}\left(v_{\vec{\omega}}, B\left(x_{0}, s\right)\right)} \\
& \leq C \prod_{i=1}^{m}\left(\left\|b_{i}\right\|_{*} \omega_{i}\left(B\left(x_{0}, s\right)\right)^{1 / p_{i}}\right) \\
& \quad \times \int_{2 s}^{\infty}\left(1+\ln \frac{r}{s}\right)^{m} \\
& \quad \times\left(\prod_{i=1}^{m}\left\|f_{i}\right\|_{L^{p_{i}\left(\omega_{i}, B\left(x_{0}, r\right)\right)}} \omega_{i}\left(B\left(x_{0}, r\right)\right)^{-1 / p_{i}}\right) \frac{d r}{r} .
\end{aligned}
$$

Proof. We will give the proof for $T_{\Pi b}$ because the proof for $T_{\Sigma b}$ is very similar but easier. Moreover, for simplicity of the expansion, we only present the case $m=2$.
We represent $f_{i}$ as $f_{i}=f_{i}^{0}+f_{i}^{\infty}$, where $f_{i}^{0}=f_{i} \chi_{B\left(x_{0}, 2 s\right)}$, $i=1,2$, and $\chi_{B\left(x_{0}, 2 s\right)}$ denotes the characteristic function of $B\left(x_{0}, 2 s\right)$. Then,

$$
\begin{aligned}
& \left\|T_{\Pi b}(\vec{f})\right\|_{L^{p}\left(v_{\vec{\omega}}, B\left(x_{0}, s\right)\right)} \\
& \leq C\left(\int_{B\left(x_{0}, s\right)}\left|T_{\Pi b}\left(f_{1}^{0}, f_{2}^{0}\right)(x)\right|^{p} \nu_{\vec{\omega}}(x) d x\right)^{1 / p} \\
& \quad+C\left(\int_{B\left(x_{0}, s\right)}\left|T_{\Pi b}\left(f_{1}^{0}, f_{2}^{\infty}\right)(x)\right|^{p} \nu_{\vec{\omega}}(x) d x\right)^{1 / p} \\
& \quad+C\left(\int_{B\left(x_{0}, s\right)}\left|T_{\Pi b}\left(f_{1}^{\infty}, f_{2}^{0}\right)(x)\right|^{p} \nu_{\vec{\omega}}(x) d x\right)^{1 / p} \\
& \quad+C\left(\int_{B\left(x_{0}, s\right)}\left|T_{\Pi b}\left(f_{1}^{\infty}, f_{2}^{\infty}\right)(x)\right|^{p} \nu_{\vec{\omega}}(x) d x\right)^{1 / p} \\
& =I+I I+I I I+I V .
\end{aligned}
$$

Since $T_{\Pi b}$ bounded from $L^{p_{1}}\left(\omega_{1}\right) \times L^{p_{2}}\left(\omega_{2}\right)$ to $L^{p}\left(\nu_{\vec{\omega}}\right)$, we get

$$
\begin{aligned}
& \left(\int_{B\left(x_{0}, s\right)}\left|T_{\Pi b}\left(f_{1}^{0}, f_{2}^{0}\right)(x)\right|^{p} v_{\vec{\omega}}(x) d x\right)^{1 / p} \\
& \quad \leq C \prod_{i=1}^{m}\left\|b_{i}\right\|_{*}\left\|f_{i}\right\|_{L^{p_{i}}\left(\omega_{i}, B\left(x_{0}, 2 s\right)\right)}
\end{aligned}
$$

Then, by (51), we get

$$
\begin{aligned}
I \leq C & \prod_{i=1}^{2}\left(\left\|b_{i}\right\|_{*} \omega_{i}\left(B\left(x_{0}, s\right)\right)^{1 / p_{i}}\right) \\
& \cdot \int_{2 s}^{\infty}\left(\prod_{i=1}^{2}\left\|f_{i}\right\|_{L^{p_{i}}\left(\omega_{i}, B\left(x_{0}, r\right)\right)} \omega_{i}\left(B\left(x_{0}, r\right)\right)^{-1 / p_{i}}\right) \frac{d r}{r} .
\end{aligned}
$$

Owing to the symmetry of $I I$ and $I I I$, we only estimate $I I$. Taking $\lambda_{i}=\left(b_{i}\right)_{B\left(x_{0}, s\right)}$, then

$$
\begin{aligned}
T_{\Pi b}( & \left.f_{1}^{0}, f_{2}^{\infty}\right)(x) \\
= & \left(b_{1}(x)-\lambda_{1}\right)\left(b_{2}(x)-\lambda_{2}\right) T\left(f_{1}^{0}, f_{2}^{\infty}\right)(x) \\
& -\left(b_{1}(x)-\lambda_{1}\right) T\left(f_{1}^{0},\left(b_{2}-\lambda_{2}\right) f_{2}^{\infty}\right)(x) \\
& -\left(b_{2}(x)-\lambda_{2}\right) T\left(\left(b_{1}-\lambda_{1}\right) f_{1}^{0}, f_{2}^{\infty}\right)(x) \\
& +T\left(\left(b_{1}-\lambda_{1}\right) f_{1}^{0},\left(b_{2}-\lambda_{2}\right) f_{2}^{\infty}\right)(x) \\
= & I I_{1}+I I_{2}+I I_{3}+I I_{4} .
\end{aligned}
$$

Similar to the estimate of (59), for any $x \in B\left(x_{0}, s\right)$, we can deduce

$$
\begin{aligned}
& \sup _{x \in B\left(x_{0}, s\right)}\left|T\left(f_{1}^{0}, f_{2}^{\infty}\right)(x)\right| \\
& \leq C \int_{2 s}^{\infty}\left(\prod_{i=1}^{2}\left\|f_{i}\right\|_{L^{p_{i}}\left(\omega_{i}, B\left(x_{0}, r\right)\right)} \omega_{i}\left(B\left(x_{0}, r\right)\right)^{-1 / p_{i}}\right) \frac{d r}{r} .
\end{aligned}
$$


By Lemma 8, we know that $\nu_{\vec{\omega}} \in A_{\infty}$. Applying Hölder's inequality and Lemma 10, we have

$$
\begin{aligned}
& \left(\int_{B\left(x_{0}, s\right)}\left|\left(b_{1}(x)-\lambda_{1}\right)\left(b_{2}(x)-\lambda_{2}\right)\right|^{p} v_{\vec{\omega}}(x) d x\right)^{1 / p} \\
& \quad \leq C \prod_{i=1}^{2}\left(\int_{B\left(x_{0}, s\right)}\left|b_{i}(x)-\lambda_{i}\right|^{2 p} v_{\vec{\omega}}(x) d x\right)^{1 / 2 p} \\
& \quad \leq C \prod_{i=1}^{2}\left\|b_{i}\right\|_{*} \cdot\left(v_{\vec{\omega}}\left(B\left(x_{0}, s\right)\right)\right)^{1 / p} .
\end{aligned}
$$

Then, by (77), (78), and (57), we have

$$
\begin{aligned}
& \left(\int_{B\left(x_{0}, s\right)}\left|I I_{1}\right|^{p} v_{\vec{\omega}}(x) d x\right)^{1 / p} \\
& \leq\left(\int_{B\left(x_{0}, s\right)}\left|\left(b_{1}(x)-\lambda_{1}\right)\left(b_{2}(x)-\lambda_{2}\right)\right|^{p} v_{\vec{\omega}}(x) d x\right)^{1 / p} \\
& \times \sup _{x \in B\left(x_{0}, s\right)}\left|T\left(f_{1}^{0}, f_{2}^{\infty}\right)(x)\right| \\
& \leq C \prod_{i=1}^{2}\left(\left\|b_{i}\right\|_{*} \omega_{i}\left(B\left(x_{0}, s\right)\right)^{1 / p_{i}}\right) \\
& \cdot \int_{2 s}^{\infty}\left(\prod_{i=1}^{2}\left\|f_{i}\right\|_{L^{p_{i}}\left(\omega_{i}, B\left(x_{0}, r\right)\right)} \omega_{i}\left(B\left(x_{0}, r\right)\right)^{-1 / p_{i}}\right) \frac{d r}{r} .
\end{aligned}
$$

Applying the size condition, we deduce that, for any $x \in$ $B\left(x_{0}, s\right)$,

$$
\begin{aligned}
& \quad\left|T\left(f_{1}^{0},\left(b_{2}-\lambda_{2}\right) f_{2}^{\infty}\right)(x)\right| \\
& \leq C \int_{B\left(x_{0}, 2 s\right)} \int_{\mathbb{R}^{n} \backslash B\left(x_{0}, 2 s\right)}\left(\left|f_{1}\left(y_{1}\right)\left(b_{2}\left(y_{2}\right)-\lambda_{2}\right) f_{2}\left(y_{2}\right)\right|\right) \\
& \quad \times\left(\left(\left|x-y_{1}\right|+\left|x-y_{2}\right|\right)^{2 n}\right)^{-1} d y_{1} d y_{2} \\
& \leq C \sum_{j=1}^{\infty}\left(2^{j+1} s\right)^{-2 n} \int_{B\left(x_{0}, 2^{j+1} s\right)}\left|f_{1}\left(y_{1}\right)\right| d y_{1} \\
& \quad \times \int_{B\left(x_{0}, 2^{j+1} s\right)}\left|\left(b_{2}\left(y_{2}\right)-\lambda_{2}\right) f_{2}\left(y_{2}\right)\right| d y_{2} .
\end{aligned}
$$

Note that

$$
\begin{aligned}
& \int_{B\left(x_{0}, 2^{j+1} s\right)}\left|f_{1}\left(y_{1}\right)\right| d y_{1} \\
& \leq C\left\|f_{1}\right\|_{L^{p_{1}\left(\omega_{1}, B\left(x_{0}, 2^{j+1} s\right)\right)}}\left\|\omega_{1}^{-1 / p_{1}}\right\|_{L^{p_{1}^{\prime}}\left(B\left(x_{0}, 2^{j+1} s\right)\right)^{\prime}} \\
& \int_{B\left(x_{0}, 2^{j+1} s\right)}\left|\left(b_{2}\left(y_{2}\right)-\lambda_{2}\right) f_{2}\left(y_{2}\right)\right| d y_{2} \\
& \leq C\left\|f_{2}\right\|_{L^{p_{2}}\left(\omega_{2}, B\left(x_{0}, 2^{k+1} s\right)\right)}\left\|b_{2}(\cdot)-\lambda_{2}\right\|_{L^{p_{2}^{\prime}}\left(\omega_{2}^{\left.1-p_{2}^{\prime}, B\left(x_{0}, 2^{k+1} s\right)\right)}\right.} .
\end{aligned}
$$

Then,

$$
\begin{aligned}
& \sup _{x \in B\left(x_{0}, s\right)}\left|T\left(f_{1}^{0},\left(b_{2}-\lambda_{2}\right) f_{2}^{\infty}\right)(x)\right| \\
& \leq C \int_{2 s}^{\infty} \prod_{i=1}^{2}\left\|f_{i}\right\|_{L^{p_{i}}\left(\omega_{i}, B\left(x_{0}, r\right)\right)}
\end{aligned}
$$

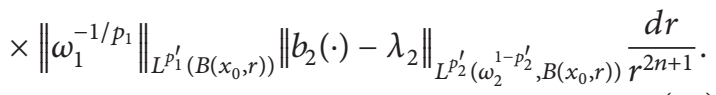

Since $\omega_{2}^{1-p_{2}^{\prime}} \in A_{2 p_{2}^{\prime}}$, then, from Lemma 12, we get

$$
\begin{aligned}
& \left\|b_{2}(\cdot)-\lambda_{2}\right\|_{L^{p_{2}^{\prime}}\left(\omega_{2}^{1-p_{2}^{\prime}}, B\left(x_{0}, r\right)\right)} \\
& \quad \leq C\left(\int_{B\left(x_{0}, r\right)}\left|b_{2}(z)-\lambda_{2}\right|^{p_{2}^{\prime}} \omega_{2}^{1-p_{2}^{\prime}}(z) d z\right)^{1 / p_{2}^{\prime}} \\
& \quad \leq C\left(1+\left|\ln \frac{r}{s}\right|\right)\left\|b_{2}\right\|_{*}\left(\omega_{2}^{1-p_{2}^{\prime}}\left(B\left(x_{0}, r\right)\right)\right)^{1 / p_{2}^{\prime}} \\
& \quad=C\left(1+\left|\ln \frac{r}{s}\right|\right)\left\|b_{2}\right\|_{*}\left\|\omega_{2}^{-1 / p_{2}}\right\|_{L^{p_{2}^{\prime}\left(B\left(x_{0}, r\right)\right)}} .
\end{aligned}
$$

By (50), we have

$$
\begin{aligned}
& \prod_{i=1}^{2}\left\|\omega_{i}^{-1 / p_{i}}\right\|_{L^{p_{i}^{\prime}}\left(B\left(x_{0}, r\right)\right)} \\
& \quad \leq C\left|B\left(x_{0}, r\right)\right|^{2} \prod_{i=1}^{2} \omega_{i}\left(B\left(x_{0}, r\right)\right)^{-1 / p_{i}} .
\end{aligned}
$$

From (82), (83), and (84), we can deduce

$$
\begin{aligned}
\sup _{x \in B\left(x_{0}, s\right)}\left|T\left(f_{1}^{0},\left(b_{2}-\lambda_{2}\right) f_{2}^{\infty}\right)(x)\right| \\
\leq C\left\|b_{2}\right\|_{*} \int_{2 s}^{\infty}\left(1+\ln \frac{r}{s}\right) \\
\quad \times\left(\prod_{i=1}^{2}\left\|f_{i}\right\|_{L^{p_{i}}\left(\omega_{i}, B\left(x_{0}, r\right)\right)} \omega_{i}\left(B\left(x_{0}, r\right)\right)^{-1 / p_{i}}\right) \frac{d r}{r} .
\end{aligned}
$$

Applying Lemma 10 and (57), we have

$$
\begin{gathered}
\left(\int_{B\left(x_{0}, s\right)}\left|b_{1}(x)-\lambda_{1}\right|^{p} \nu_{\vec{\omega}}(x) d x\right)^{1 / p} \\
\leq C\left\|b_{1}\right\|_{*}\left(v_{\vec{\omega}}\left(B\left(x_{0}, s\right)\right)\right)^{1 / p} \\
\leq C\left\|b_{1}\right\|_{*} \prod_{i=1}^{2} \omega_{i}\left(B\left(x_{0}, r\right)\right)^{1 / p_{i}} .
\end{gathered}
$$


Then, by (85),

$$
\begin{aligned}
& \left(\int_{B\left(x_{0}, s\right)}\left|I I_{2}\right|^{p} \nu_{\vec{\omega}}(x) d x\right)^{1 / p} \\
& \leq\left(\int_{B\left(x_{0}, s\right)}\left|b_{1}(x)-\lambda_{1}\right|^{p} \nu_{\vec{\omega}}(x) d x\right)^{1 / p} \\
& \quad \times \sup _{x \in B\left(x_{0}, s\right)}\left|T\left(f_{1}^{0},\left(b_{2}-\lambda_{2}\right) f_{2}^{\infty}\right)(x)\right| \\
& \leq C \prod_{i=1}^{2}\left(\left\|b_{i}\right\|_{*} \omega_{i}\left(B\left(x_{0}, s\right)\right)^{1 / p_{i}}\right) \\
& \quad \times \int_{2 s}^{\infty}\left(1+\ln \frac{r}{s}\right) \\
& \quad \times\left(\prod_{i=1}^{2}\left\|f_{i}\right\|_{L^{p_{i}}\left(\omega_{i}, B\left(x_{0}, r\right)\right)} \omega_{i}\left(B\left(x_{0}, r\right)\right)^{-1 / p_{i}}\right) \frac{d r}{r} .
\end{aligned}
$$

Similarly, we also have that

$$
\begin{aligned}
& \left(\int_{B\left(x_{0}, s\right)}\left|I I_{3}\right|^{p} \nu_{\vec{\omega}}(x) d x\right)^{1 / p} \\
& \leq C \prod_{i=1}^{2}\left(\left\|b_{i}\right\|_{*} \omega_{i}\left(B\left(x_{0}, s\right)\right)^{1 / p_{i}}\right) \\
& \quad \times \int_{2 s}^{\infty}\left(1+\ln \frac{r}{s}\right) \\
& \quad \times\left(\prod_{i=1}^{2}\left\|f_{i}\right\|_{L^{p_{i}\left(\omega_{i}, B\left(x_{0}, r\right)\right)}} \omega_{i}\left(B\left(x_{0}, r\right)\right)^{-1 / p_{i}}\right) \frac{d r}{r} .
\end{aligned}
$$

Applying the size condition again, for any $x \in B\left(x_{0}, s\right)$, with the same method of estimate for (85), we have

$$
\begin{aligned}
& \left|T\left(\left(b_{1}-\lambda_{1}\right) f_{1}^{0},\left(b_{2}-\lambda_{2}\right) f_{2}^{\infty}\right)(x)\right| \\
& \leq C \sum_{j=1}^{\infty}\left(2^{j+1} s\right)^{-2 n} \prod_{i=1}^{2} \int_{B\left(x_{0}, 2^{j+1} s\right)}\left|\left(b_{i}\left(y_{i}\right)-\lambda_{i}\right) f_{i}\left(y_{i}\right)\right| d y_{i} \\
& \leq C \int_{2 s}^{\infty}\left(\prod_{i=1}^{2}\left\|f_{i}\right\|_{L^{p_{i}}\left(\omega_{i}, B\left(x_{0}, r\right)\right)}\right) \frac{d r}{r^{2 n+1}} \\
& \left.\leq C \prod_{i=1}^{2}\left\|b_{i}\right\|_{*}\left\|b_{i}(\cdot)-\lambda_{i}\right\|_{L^{p_{i}^{\prime}}\left(\omega_{i}^{1-p_{i}^{\prime}}, B\left(x_{0}, r\right)\right)}\right) \\
& \cdot \int_{2 s}^{\infty}\left(1+\ln \frac{r}{s}\right)^{2} \\
& \quad \times\left(\prod_{i=1}^{2}\left\|f_{i}\right\|_{L^{p_{i}}\left(\omega_{i}, B\left(x_{0}, r\right)\right)} \omega_{i}\left(B\left(x_{0}, r\right)\right)^{-1 / p_{i}}\right) \frac{d r}{r} .
\end{aligned}
$$

Then,

$$
\begin{aligned}
& \left(\int_{B\left(x_{0}, s\right)}\left|I I_{4}\right|^{p} \nu_{\vec{\omega}}(x) d x\right)^{1 / p} \\
& \leq C\left(\nu_{\vec{\omega}}\left(B\left(x_{0}, s\right)\right)\right)^{1 / p} \\
& \quad \times \sup _{x \in B\left(x_{0}, s\right)}\left|\left(\left(b_{1}-\lambda_{1}\right) f_{1}^{0},\left(b_{2}-\lambda_{2}\right) f_{2}^{\infty}\right)(x)\right| \\
& \leq C \prod_{i=1}^{2}\left(\left\|b_{i}\right\|_{*} \omega_{i}\left(B\left(x_{0}, s\right)\right)^{1 / p_{i}}\right) \\
& \quad \cdot \int_{2 s}^{\infty}\left(1+\ln \frac{r}{s}\right)^{2} \\
& \quad \times\left(\prod_{i=1}^{2}\left\|f_{i}\right\|_{L^{p_{i}}\left(\omega_{i}, B\left(x_{0}, r\right)\right)} \omega_{i}\left(B\left(x_{0}, r\right)\right)^{-1 / p_{i}}\right) \frac{d r}{r} .
\end{aligned}
$$

Then, combining (79), (87), (88), and (90), we get

$$
\begin{aligned}
& \left(\int_{B\left(x_{0}, s\right)}|I I|^{p} v_{\vec{\omega}}(x) d x\right)^{1 / p} \\
& \leq C \prod_{i=1}^{2}\left(\left\|b_{i}\right\|_{*} \omega_{i}\left(B\left(x_{0}, s\right)\right)^{1 / p_{i}}\right) \\
& \quad \times \int_{2 s}^{\infty}\left(1+\ln \frac{r}{s}\right)^{2} \\
& \quad \times\left(\prod_{i=1}^{2}\left\|f_{i}\right\|_{L^{p_{i}}\left(\omega_{i}, B\left(x_{0}, r\right)\right)} \omega_{i}\left(B\left(x_{0}, r\right)\right)^{-1 / p_{i}}\right) \frac{d r}{r} .
\end{aligned}
$$

Finally, we still decompose $T_{\Pi b}\left(f_{1}^{\infty}, f_{2}^{\infty}\right)(x)$ as follows:

$$
\begin{aligned}
T_{\Pi b} & \left(f_{1}^{\infty}, f_{2}^{\infty}\right)(x) \\
= & \left(b_{1}(x)-\lambda_{1}\right)\left(b_{2}(x)-\lambda_{2}\right) T\left(f_{1}^{\infty}, f_{2}^{\infty}\right)(x) \\
& -\left(b_{1}(x)-\lambda_{1}\right) T\left(f_{1}^{\infty},\left(b_{2}-\lambda_{2}\right) f_{2}^{\infty}\right)(x) \\
& -\left(b_{2}(x)-\lambda_{2}\right) T\left(\left(b_{1}-\lambda_{1}\right) f_{1}^{\infty}, f_{2}^{\infty}\right)(x) \\
& +T\left(\left(b_{1}-\lambda_{1}\right) f_{1}^{\infty},\left(b_{2}-\lambda_{2}\right) f_{2}^{\infty}\right)(x) \\
= & I V_{1}+I V_{2}+I V_{3}+I V_{4} .
\end{aligned}
$$

Because each term $I V_{j}$ is completely analogous to $I I_{j}, j=$ $1,2,3,4$ with a bit difference, we get the following estimate without details:

$$
\begin{aligned}
& \left(\int_{B\left(x_{0}, s\right)}|I V|^{p} \nu_{\vec{\omega}}(x) d x\right)^{1 / p} \\
& \leq C \prod_{i=1}^{2}\left(\left\|b_{i}\right\|_{*} \omega_{i}\left(B\left(x_{0}, s\right)\right)^{1 / p_{i}}\right) \\
& \quad \times \int_{2 s}^{\infty}\left(1+\ln \frac{r}{s}\right)^{2} \\
& \quad \times\left(\prod_{i=1}^{2}\left\|f_{i}\right\|_{L^{p_{i}\left(\omega_{i}, B\left(x_{0}, r\right)\right)}} \omega_{i}\left(B\left(x_{0}, r\right)\right)^{-1 / p_{i}}\right) \frac{d r}{r} .
\end{aligned}
$$

Summing up the above estimates, (72) is proved. 
We give the proof of Theorem 3 in the following. From (62) and (72),

$$
\begin{aligned}
& \left(\frac{1}{v_{\vec{\omega}}(B(x, s))} \int_{B(x, s)}\left|T_{\Pi b}(\vec{f})(y)\right|^{p} \nu_{\vec{\omega}}(y) d y\right)^{1 / p} \\
& \leq C \prod_{i=1}^{m}\|b\|_{*} \\
& \quad \cdot \int_{s}^{\infty}\left(1+\ln \frac{r}{s}\right)^{m} \\
& \quad \times\left(\prod_{i=1}^{m}\left\|f_{i}\right\|_{L^{p_{i}\left(\omega_{i}, B(x, r)\right)}} \omega_{i}(B(x, r))^{-1 / p_{i}}\right) \frac{d r}{r} .
\end{aligned}
$$

Since $\left(\vec{\varphi}_{1}, \vec{\varphi}_{2}\right)$ satisfies condition (15), $\left\|f_{i}\right\|_{L^{p_{i}(B(x, r))}}$ are all nondecreasing function of $r$, and $f_{i} \in M_{\varphi_{1 i}}^{p_{i}}\left(\omega_{i}\right)$, then

$$
\begin{aligned}
\int_{s}^{\infty}\left(1+\ln \frac{r}{s}\right)^{m}\left(\prod_{i=1}^{m}\left\|f_{i}\right\|_{L^{p_{i}}\left(\omega_{i}, B(x, r)\right)} \omega_{i}(B(x, r))^{-1 / p_{i}}\right) \frac{d r}{r} \\
=\int_{s}^{\infty} \frac{\prod_{i=1}^{m}\left\|f_{i}\right\|_{L^{p_{i}}\left(\omega_{i}, B(x, r)\right)}}{\operatorname{ess}_{\inf _{r<t<\infty} \prod_{i=1}^{m} \varphi_{1 i}(x, t) \omega_{i}(B(x, t))^{1 / p_{i}}}} \\
\quad \times\left(1+\ln \frac{r}{s}\right)^{m} \\
\quad \times \frac{\operatorname{ess} \inf _{r<t<\infty} \prod_{i=1}^{m} \varphi_{1 i}(x, t) \omega_{i}(B(x, t))^{1 / p_{i}}}{\prod_{i=1}^{n} \omega_{i}(B(x, t))^{1 / p_{i}}} \frac{d r}{r} \\
\leq C \prod_{i=1}^{m}\left\|f_{i}\right\|_{M_{\varphi_{1 i}}^{p_{i}}\left(\omega_{i}\right)} \\
\quad \times \int_{s}^{\infty}\left(1+\ln \frac{r}{s}\right)^{m} \\
\leq C \varphi_{2}(x, s) \prod_{i=1}^{m}\left\|f_{i}\right\|_{M_{\varphi_{1 i}}^{p_{i}}\left(\omega_{i}\right)} \\
\quad \times \frac{\operatorname{ess~inf}_{r<t<\infty} \prod_{i=1}^{m} \varphi_{1 i}(x, t) \omega_{i}(B(x, t))^{1 / p_{i}}}{\prod_{i=1}^{n} \omega_{i}(B(x, t))^{1 / p_{i}}} \frac{d r}{r}
\end{aligned}
$$

Combining (94) and (95), we have proved that

$$
\left\|T_{\Pi b}(\vec{f})\right\|_{M_{\varphi_{2}}^{p}\left(v_{\vec{\omega}}\right)} \leq C \prod_{i=1}^{m}\left\|b_{i}\right\|_{*}\left\|f_{i}\right\|_{M_{\varphi_{1 i}}^{p_{i}}\left(\omega_{i}\right)} .
$$

\section{Conflict of Interests}

The authors declare that there is no conflict of interests regarding the publication of this paper.

\section{Acknowledgments}

The authors are grateful to the anonymous reviewers and the editor for their several comments and suggestions which contributed to the improvement of this paper. The project is supported by the National Natural Science Foundation of China (no. 61340014).

\section{References}

[1] R. R. Coifman and Y. Meyer, "On commutators of singular integrals and bilinear singular integrals," Transactions of the American Mathematical Society, vol. 212, pp. 315-331, 1975.

[2] L. Grafakos and R. H. Torres, "Multilinear Calderón-Zygmund theory," Advances in Mathematics, vol. 165, no. 1, pp. 124-164, 2002.

[3] L. Grafakos and R. H. Torres, "Maximal operator and weighted norm inequalities for multilinear singular integrals," Indiana University Mathematics Journal, vol. 51, no. 5, pp. 1261-1276, 2002.

[4] L. Grafakos and R. Torres, "On multilinear singular integrals of Calderón-Zygmund type," Publicacions Matemàtiques, vol. 46, pp. 57-91, 2002.

[5] A. K. Lerner, S. Ombrosi, C. Pérez, R. H. Torres, and R. TrujilloGonzález, "New maximal functions and multiple weights for the multilinear Calderón-Zygmund theory," Advances in Mathematics, vol. 220, no. 4, pp. 1222-1264, 2009.

[6] H. Wang and W. Yi, "Multilinear singular and fractional integral operators on weighted Morrey spaces," Journal of Function Spaces and Applications, Article ID 735795, pp. 1-11, 2013.

[7] S. Wang and Y. Jiang, "Multilinear singular integrals and their commutators with nonsmooth kernels on weighted Morrey spaces," Abstract and Applied Analysis, vol. 2013, Article ID 280401, 10 pages, 2013.

[8] C. B. Morrey, "On the solutions of quasi-linear elliptic partial differential equations," Transactions of the American Mathematical Society, vol. 43, no. 1, pp. 126-166, 1938.

[9] F. Chiarenza and M. Frasca, "Morrey spaces and HardyLittlewood maximal function," Rendiconti di Matematica e delle sue Applicazioni, vol. 7, no. 3-4, pp. 273-279, 1987.

[10] G. di Fazio and M. A. Ragusa, "Interior estimates in Morrey spaces for strong solutions to nondivergence form equations with discontinuous coefficients," Journal of Functional Analysis, vol. 112, no. 2, pp. 241-256, 1993.

[11] J. Peetre, "On the theory of $\mathscr{L}_{p, \lambda}$ spaces," Journal of Functional Analysis, vol. 4, no. 1, pp. 71-87, 1969.

[12] T. Mizuhara, "Boundedness of some classical operators on generalized Morrey spaces," in ICM-90 Satellite Conference Proceedings, pp. 183-189, Springer, Tokyo, Japan, 1991.

[13] Y. Komori and S. Shirai, "Weighted Morrey spaces and a singular integral operator," Mathematische Nachrichten, vol. 282, no. 2, pp. 219-231, 2009.

[14] V. Guliyev, "Generalized weighted Morrey spaces and higher order commutators of sublinear operators," Eurasian Mathematical Journal, vol. 3, no. 3, pp. 33-61, 2012.

[15] V. S. Guliyev, S. S. Aliyev, T. Karaman, and P. S. Shukurov, "Boundedness of sublinear operators and commutators on generalized Morrey spaces," Integral Equations and Operator Theory, vol. 71, no. 3, pp. 327-355, 2011.

[16] V. S. Guliyev, S. S. Aliyev, and T. Karaman, "Boundedness of a class of sublinear operators and their commutators on generalized Morrey spaces," Abstract and Applied Analysis, vol. 2011, Article ID 356041, 18 pages, 2011. 
[17] C. Pérez, G. Pradolini, R. Torres, and R. Trujillo-Gonzalez, "End-point estimates for iterated commutators for multilinear singular integrals," Bulletin London Mathematical Society, 2013.

[18] R. L. Wheeden and A. Zygmund, Measure and Integral, An Introduction to Real Analysis, vol. 43 of Pure and Applied Mathematics, Marcel Dekker, New York, NY, USA, 1977.

[19] B. Muckenhoupt, "Weighted norm inequalities for the Hardy maximal function," Transactions of the American Mathematical Society, vol. 165, pp. 207-226, 1972.

[20] J. García-Cuerva and J.-L. Rubio De Francia, Weighted Norm Inequalities and Related Topics, vol. 116 of North-Holland Mathematics Studies, North-Holland, 1985.

[21] F. John and L. Nirenberg, "On functions of bounded mean oscillation," Communications on Pure and Applied Mathematics, vol. 14, pp. 415-426, 1961.

[22] Y. Hu, Y. He, and Y. Wang, "The commutators of fractional integrals on generalized Herz spaces," Journal of Function Spaces, vol. 2014, Article ID 428493, 6 pages, 2014.

[23] Y. Lin and S. Lu, "Strongly singular Calderón-Zygmund operators and their commutators," Jordan Journal of Mathematics and Statistics, vol. 1, pp. 31-49, 2008. 


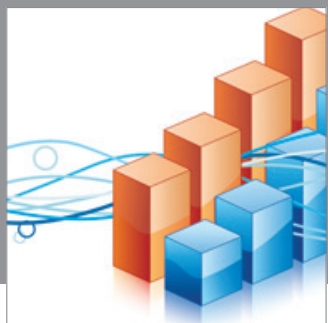

Advances in

Operations Research

mansans

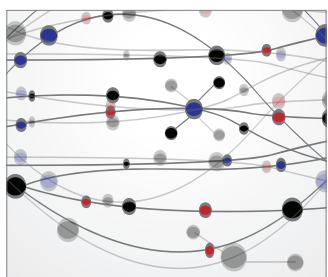

The Scientific World Journal
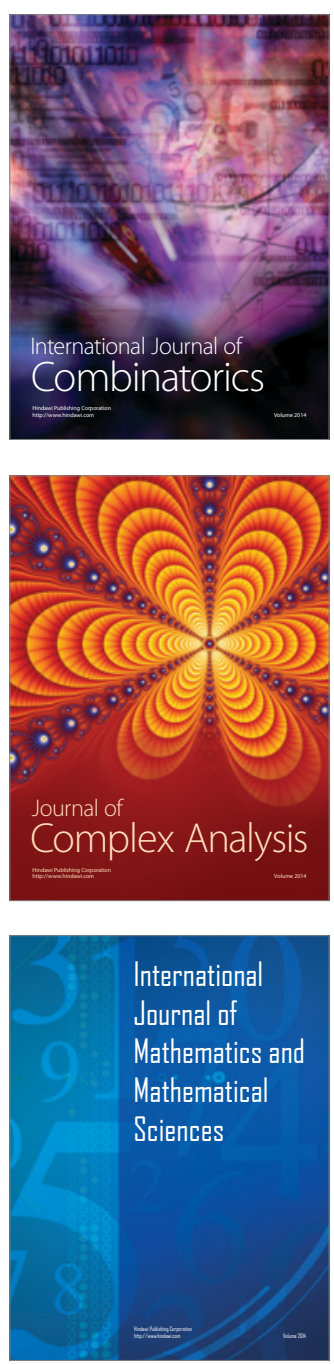
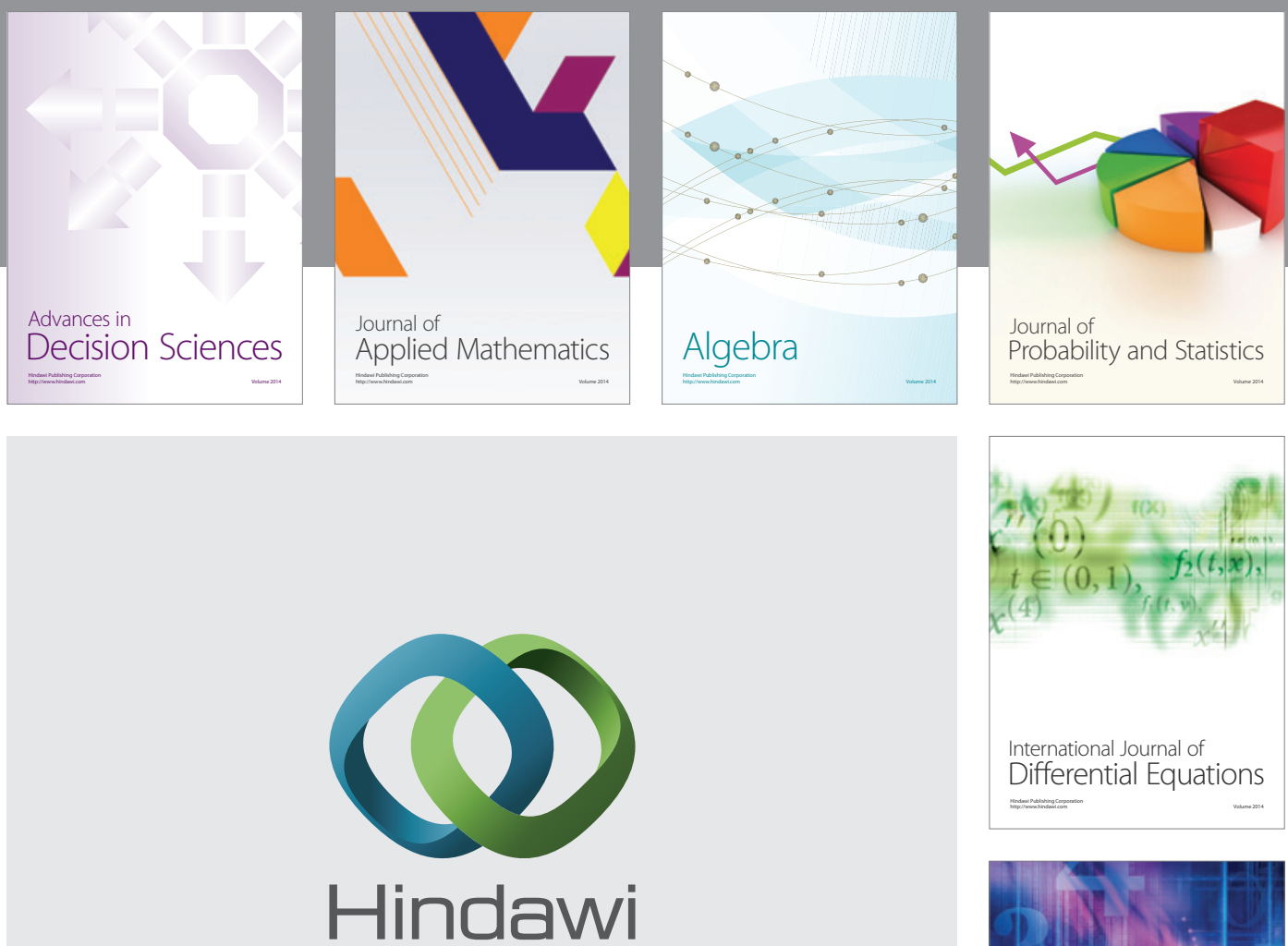

Submit your manuscripts at http://www.hindawi.com
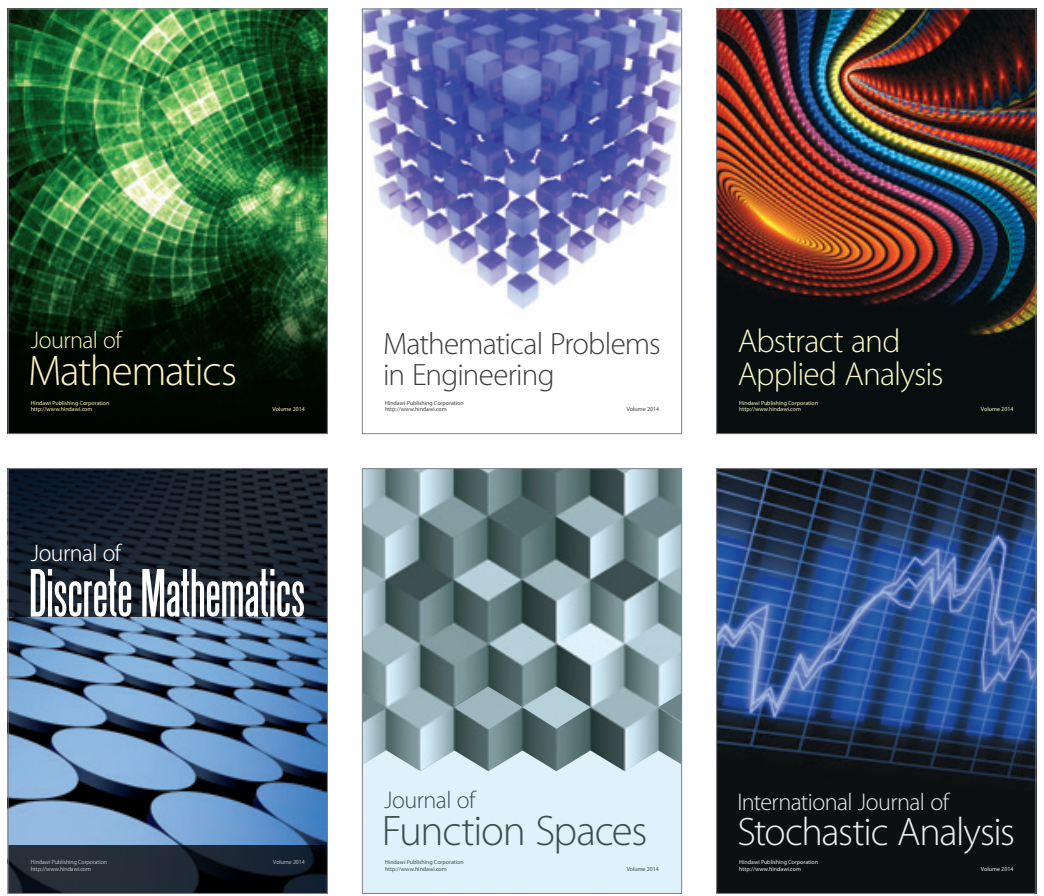

Journal of

Function Spaces

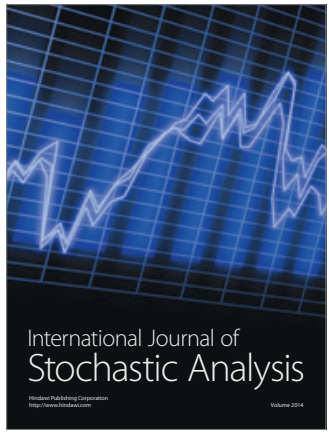

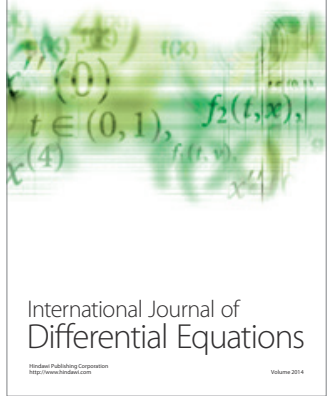
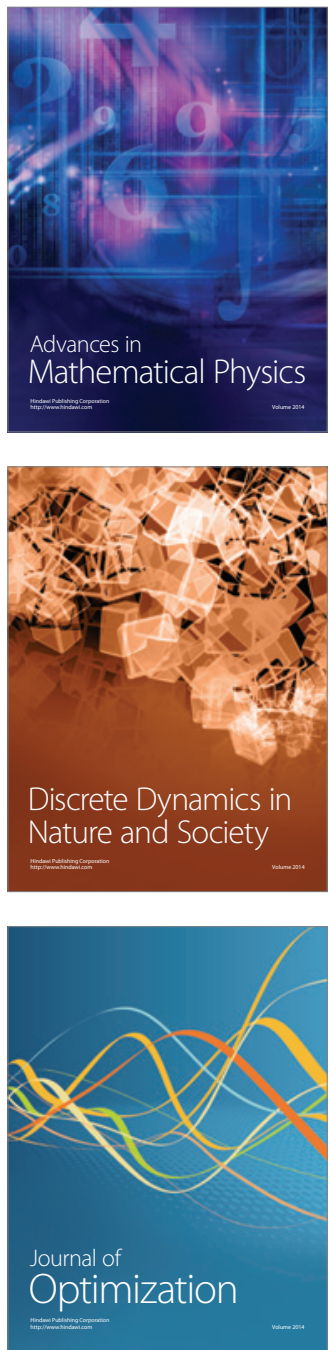